\title{
Controlling Aggregate Interference under Adjacent Channel Interference Constraint in TV White Space
}

\author{
Lei Shi, Ki Won Sung, and Jens Zander \\ KTH Royal Institute of Technology, Wireless@KTH, Stockholm, Sweden \\ E-mail: 1shi@kth.se,sungkw@kth.se,jenz@kth.se
}

\begin{abstract}
TV White space, where secondary systems can be deployed inside the TV coverage area and utilize the geographically unoccupied TV channels, is considered as a promising solution to relieve the spectrum shortage. To utilize this spectrum, the secondary users must ensure the protection of $T V$ reception from harmful interference on both co-channel and adjacent channels. In this paper, we propose an analytical approach to determining the permissible transmit power for short-range secondary users under aggregate adjacent channel interference constraint in TV white space. This approach employs statistical interference modeling which considers random deployment of secondary users, antenna gain pattern, shadow fading, and the cumulative effect of interference from multiple adjacent channels. Numerical results show that the proposed scheme permits significantly higher transmit power than the existing deterministic method does, while at the same time, it keeps the required level of TV protection. Therefore, considerable potential for the shortrange secondary access to TV white space is expected with our approach.
\end{abstract}

Index Terms - TV White Space, aggregate interference, transmit power, adjacent channel, geo-location database.

\section{INTRODUCTION}

With the rapid growth of wireless data traffic, the limited spectrum has become the bottleneck for the development of wireless services. Secondary access in the VHF/UHF TV band, often referred to as TV white space (TVWS), is considered as one promising solution to this 'spectrum scarcity' problem. It allows secondary users (SUs) to access the locally or temporally unoccupied TV channels, as long as the primary user (PU) is protected from the secondary interference. The abundance of the potential available spectrum in TVWS and its favorable propagation characteristics have attracted wide interests from academics and industry alike [1].

The regulators, including FCC in the US [2], CEPT in Europe [3] and Ofcom in the UK [4], have taken the initiative to establish methodologies for estimating the TVWS availability, in terms of the permissible secondary transmit power at different locations on different channels. To facilitate the detection of spectrum availability, it is recommended to utilize the geo-location database, which contains information such as TV coverage area, terrain elevation, population density, and etc.

Previous studies in this area have mainly focused on the cochannel interference case, where the SUs are located outside the TV coverage area and transmitting on the same channel used by the TV broadcasting system [5] [6]. These analysis have extended the secondary transmit power allocation problem to multiple SU case, with either statistical or deterministic interference constraints.

Due to the imperfection of the TV receiver filter, however, the TV reception is subjected to not only co-channel interference (CCI) but also adjacent channel interference (ACI). Thus, even the SUs transmitting on non-broadcasting channels may still cause harmful interference to TV receivers near by. This is particularly true for the 'WiFi-like' or 'femtocell-like' short-range SUs that can be deployed with high density, as the cumulative effect of ACI over the spectral domain [7] could cause significant deterioration to the TV reception quality. However, unlike the CCI case where the geo-location database can be used to estimate the interfering link gain, it is very difficult to determine the ACI link gain between the SU and the victim TV receiver in its proximity, due to the uncertainty of the TV receiver location.

One deterministic approach, called 'Reference Geometry', was proposed in ECC report 159 [3] to address the ACI issue. Its basic concept is to define certain fixed ACI link geometries corresponding to the worst case scenario for different types of PU/SU deployments. In [8], it is proposed to extend this method to include multiple SUs case by placing $3 \mathrm{SUs}$ at the same reference distance. While this deterministic method is straightforward to implement, its inflexibility also leads to pessimistic estimation of the permissible transmit power on adjacent channels [9].

Motivated by these facts, we propose a statistical approach to determine the permissible secondary transmit power under aggregate ACI constraint, exploiting the specific features of TVWS, such as the random deployment of SUs, TV receiver antenna directivity [10], and the cumulative effect of ACI. The proposed method utilizes the information available in the geolocation database to estimate the permissible transmit power for different SUs, under PU protection constraint.

In the following of this paper, we will start by briefly explaining the system models in Section II. Then the permissible transmit power problem is described in Section III. Numerical results are shown in Section IV. Finally conclusions are drawn in Section V.

\section{SySTEM MODEL}

\section{A. TV Coverage}

Let us assume a TV transmitter broadcasting on a set of channels, $X$. The studied area is divided into small area ele- 


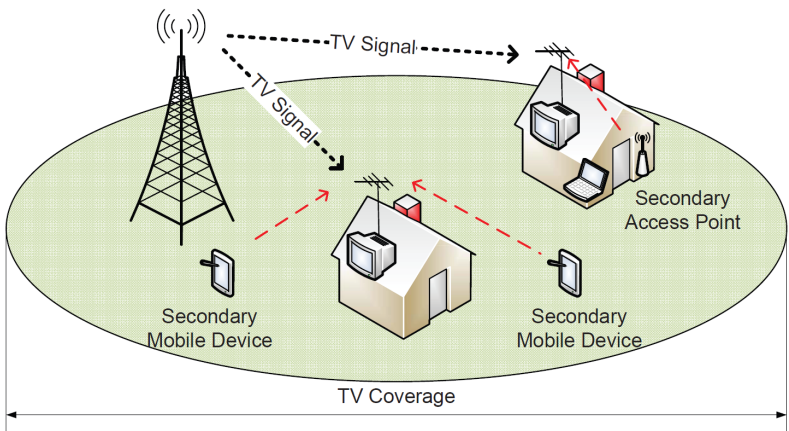

Fig. 1: System model for secondary access in TVWS.

ments according to the limited resolution of the geo-location database, denoted as 'pixels'. In pixel $i$, all TV receivers are assumed to have approximately the same received TV signal strength $P_{t v}^{i}$. The minimum TV receiver sensitivity level is $P_{t v}^{\min }$.

The measure for TV coverage quality is the location probability, defined as the chance of successful reception of any TV in that pixel. Unsuccessful TV reception is termed outage, either due to the TV signal fading or other interferences. For a TV in pixel $i$, the location probability without secondary interference is designated $q_{1}^{i}$

$$
q_{1}^{i}=\operatorname{Pr}\left\{P_{t v}^{i} \geq P_{t v}^{\min }+\gamma_{t v} I_{t v}^{i}\right\}
$$

where, $\gamma_{t v}$ is the required minimum ratio between TV signal and TV self-interference, and $I_{t v}^{i}$ is the received TV selfinterference power from other TV transmitters. The coverage area is defined by $q_{1}^{i} \geq q^{*}$, with $q^{*}$ being the minimum required location probability defined by the regulator. The set of pixels inside the coverage of channels $x \in X$ is denoted as $\Lambda_{X}^{t v}$.

\section{B. Interference from Secondary Users}

To emphasize the cumulative effect of ACI, we consider a 'WiFi-like' or 'femtocell-like' short-range secondary system deployed inside the TV coverage area following spatial Poisson processes with density $\lambda$. The secondary transmit power is assumed to be limited by the aggregate adjacent channel interference constraint, due to their close distance to the potential victim TV receiver.

Inside $\Lambda_{X}^{t v}$, the SUs can access to the unoccupied channels, $y \in Y: Y=X^{c}$ (where $X^{c}$ is the complement of $X$, with all the channels in VHF/UHF band as the universal set). Assuming the $\mathrm{SU}$ is transmitting with power level $P_{s u}^{y}$ on channel $y$, the interference received by a TV on channel $x$ in pixel $i$ can be written as

$$
I_{s u}^{x}=P_{s u}^{y} g_{f} g_{\theta}(\theta) g_{r}(d) .
$$

Here $g_{f}$ is the channel fading random variable. $g_{\theta}(\theta)$ and $g_{r}(d)$ are the TV receiver antenna gain and the distance dependent pathloss between the SU and the TV receiver, respectively. Note that $d$ is generally unknown and can only be

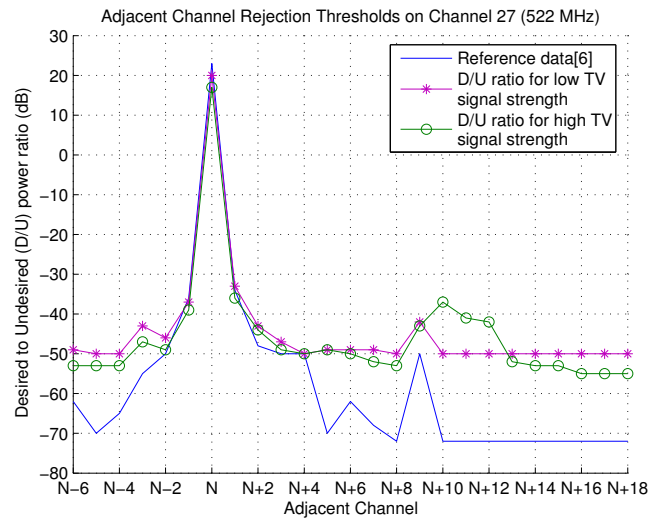

Fig. 2: Protection ratio as a function of frequency offset between TV signal and interfering signal [7].

estimated based on SU density, due to the uncertainty of TV receiver location. Therefore, any TV in the same pixel on the same channel would experience the same level of interference statistically.

\section{Cumulative Effect of Multiple Adjacent Channels Interfer- ences}

It has been reported in [7] that, the permissible secondary transmit power in a given adjacent channel must be reduced, when more adjacent channels are accessed by SUs simultaneously. It indicates the cumulative effect of interferences from multiple adjacent channels.

To model this effect, we first define an equivalent $\mathrm{CCI}$ $\left(\bar{I}_{s u, a}^{x}\right)$ on TV broadcasting channel $x$, that would cause the same level of damage to the TV reception as the aggregate ACI from interferers on the neighboring channels $y_{n} \in Y$. It can be approximated by the linear summation of ACIs, weighted by the protection ratio of each channel respectively

$\bar{I}_{s u, a}^{x}=\sum_{n=1}^{N} \frac{\gamma_{s u}\left(\Delta f_{x-y_{n}}\right)}{\gamma_{s u}(\Delta 0)} I_{s u, n}^{x}=\sum_{n=1}^{N} \frac{\gamma_{s u}\left(\Delta f_{x-y_{n}}\right)}{\gamma_{s u}(\Delta 0)} P_{s u}^{y_{n}} G_{n}$,

where $\gamma_{s u}(\Delta f)$ is the minimum required $\mathrm{TV}$ signal to $\mathrm{SU}$ interference ratio with frequency offset of $\Delta f$ (also known as protection ratio; see Fig. 2). $G_{n}=g_{f} g_{\theta}\left(\theta_{n}\right) g_{r}\left(d_{n}\right)$ denotes the coupling gain of the $n^{\text {th }}$ interfering link from adjacent channels. $N$ is the total number of active SUs.

Without loss of generality, we can assume the aggregate interference received by all TV receivers in the same pixel have the same statistical properties. We further assume that, as the number of active SUs increases, the transmit power of SUs on different channels must be decreased with equal proportion, to satisfy the aggregate interference constraint as defined in the following section.

\section{PERmissible Transmit Power Under Adjacent CHANNEL INTERFERENCE CONSTRAINT}

The permissible transmit power $P_{s u}^{i, y *}$ must be determined per pixel $i$ and TVWS channel $y$ based on the information 
available in the geo-location database, to ensure the reduced location probability $q_{2}^{i, x}$ under secondary interference is no less than $q *$ :

$$
\begin{aligned}
q_{2}^{i, x} & =\operatorname{Pr}\left\{P_{t v}^{i, x} \geq P_{t v}^{m i n}+\gamma_{t v} I_{t v}^{i, x}+\gamma_{s u}(\Delta 0) \bar{I}_{s u, a}^{i, x}\right\} \\
& =\operatorname{Pr}\left\{P_{t v}^{i, x} \geq P_{t v}^{m i n}+\gamma_{t v} I_{t v}^{i, x}\right. \\
& \left.+\sum_{y \in Y} \gamma\left(\Delta f_{x-y}\right) \sum_{j \in \Lambda_{y}^{s u}} p_{s u}^{j, y *} \sum_{n=1}^{N_{j}^{y}} G_{n}\right\} \geq q^{*}, \\
& \forall x \in X, \forall i \in \Lambda_{X}^{t v} .
\end{aligned}
$$

Here $N_{j}^{y}$ is the number of SUs in pixel $j$ on channel $y$. From the geo-location database's perspective, these SUs share the same interference properties, therefore they would be assigned with the same permissible transmit power level.

According to (4), all SUs transmitting on the adjacent channels $y \in Y$, both inside and outside the coverage area of channel $x$ may contribute to the aggregate ACI received by a TV in pixel $i$. In practices, however, we can limit our calculation to a few pixels $j$ within the dominant interference region $j \in \Lambda_{\varepsilon}^{i}$, where the majority, $(100-\varepsilon) \%$, of the aggregate interference are generated [11]. For instance, our study has shown that, over $99.5 \%$ of the aggregate ACI in suburban environment would come from an area with radius less than 500 meters. Within this region, the differences in population densities and TV coverage qualities are almost negligible, thus we can assume that

$$
P_{s u}^{i, y *} \approx P_{s u}^{j, y *}, \forall j \in \Lambda_{\varepsilon}^{i} .
$$

Denoting the equivalent permissible transmit power on channel $y$ as $\bar{P}_{s u}^{j, x *}=P_{s u}^{j, y *} \gamma\left(\Delta f_{x-y}\right)$, the constraint in (4) can be approximated as

$$
\begin{aligned}
q_{2}^{i, x} & \approx \operatorname{Pr}\left\{P_{t v}^{i, x} \geq P_{t v}^{m i n}+\gamma_{t v} I_{t v}^{i, x}+\bar{P}_{s u}^{i, x *} \sum_{n=1}^{N_{\varepsilon}^{i}} G_{n}\right\} \\
& =\operatorname{Pr}\left\{P_{t v}^{i, x}-P_{t v}^{m i n}-\gamma_{t v} I_{t v}^{i, x} \geq \bar{P}_{s u}^{i, x *} \sum_{n=1}^{N_{\varepsilon}^{i}} G_{n}\right\} \\
& =\operatorname{Pr}\left\{\bar{P}_{s u}^{i, x *} \leq \frac{Z_{t v}^{i, x}}{\sum_{n=1}^{i} G_{n}}\right\}=\operatorname{Pr}\left\{\bar{P}_{s u}^{i, x *} \leq \frac{Z_{t v}^{i, x}}{G_{a}^{i}}\right\} \geq q^{*}, \\
& \forall x \in X, \forall i \in \Lambda_{X}^{t v},
\end{aligned}
$$

where $Z_{t v}^{i, x}=P_{t v}^{i, x}-P_{t v}^{m i n}-\gamma_{t v} I_{t v}^{i, x}$ and $G_{a}=\sum_{n=1}^{N_{\varepsilon}^{i}} G_{n}$. And $N_{\varepsilon}^{i}$ denotes the total number of active SUs transmitting on all adjacent channels inside $\Lambda_{\varepsilon}^{i}$. It follows Poisson distribution with density $\lambda^{i}$

With the simplified constraint in (6), the equivalent permissible transmit power $\bar{P}_{s u}^{i, x *}$ can be solved for each pixel $i$ and each channel $x$ separately, as long as we can find the joint distribution of $Z_{t v}^{i} / G_{a}^{i}$.

\section{A. Cumulant Based Log-normal Approximation of Secondary Interference}

Considering that the SU deployment follows Poisson spatial distribution, their aggregate interference can be approximated by different distributions, such as log-normal, shifted-lognormal or truncated-stable distribution [12] [13].

We choose the log-normal approximation, because of its easy conversion into logarithmic scale, and good approximation of the upper tail of the aggregate interference distribution. By using the first two cumulants [14] of $G_{a}^{i}$, it can be approximated by a log-normal random variable $\widehat{G}_{a}^{i}$, with the following probability distribution function (pdf):

$$
f_{G_{a}^{i}}(g) \approx f_{\widehat{G}_{a}^{i}}(\widehat{g})=\frac{1}{\widehat{g} \sigma_{\widehat{G}_{a}^{i}} \sqrt{2 \pi}} \exp \left[\frac{-\left(\ln \widehat{g}-\mu_{\widehat{G}_{a}^{i}}\right)^{2}}{2 \sigma_{\widehat{G}_{a}^{i}}^{2}}\right],
$$

where $\mu_{\widehat{G}_{a}^{i}}$ and $\sigma_{\widehat{G}_{a}^{i}}$ can be obtained by matching the cumulants of $\widehat{G}_{a}^{i}$ to that of $G_{a}^{i}$ using the following equations:

$$
\begin{gathered}
\kappa_{1}\left(G_{a}^{i}\right)=\exp \left[\mu_{\widehat{G}_{a}^{i}}+\sigma_{\widehat{G}_{a}^{i}}^{2} / 2\right], \\
\kappa_{2}\left(G_{a}^{i}\right)=\left[\exp \left(\sigma_{\widehat{G}_{a}^{i}}^{2}\right)-1\right] \exp \left(2 \mu_{\widehat{G}_{a}^{i}}+\sigma_{\widehat{G}_{a}^{i}}^{2}\right) .
\end{gathered}
$$

The cumulant $\kappa_{m}\left(G_{a}^{i}\right)$ is given by

$$
\kappa_{m}\left(G_{a}^{i}\right)=2 \pi \lambda^{i} \widetilde{\mu}_{m}\left(G_{f}\right) \widetilde{\mu}_{m}\left(G_{\theta}\right) \int_{d_{0}}^{R_{\epsilon}} g_{r, s u}^{m}(r) r d r .
$$

where $R_{\varepsilon}$ is the radius of the dominant interference region. $\widetilde{\mu}_{m}\left(G_{f}\right)$ and $\widetilde{\mu}_{m}\left(G_{\theta}\right)$ are the $m^{t h}$ raw moments of the distributions of channel fading and antenna gain, respectively. $d_{0}$ is the minimum separation distance between the TV receiver antenna and the interfering SU.

\section{B. Log-Normal Approximation of TV Signal and TV Self- interference}

Assuming shadow fading in TV signals, $Z_{t v}^{i, x}$ can be modeled as the difference between log-normal random variables and a linear constant. Recall that $q_{1}^{i, x}=\operatorname{Pr}\left\{Z_{t v}^{i, x} \geq 0\right\}$. $Z_{t v}^{i, x}$ can be negative with probability $1-q_{1}^{i, x}$, thus it cannot be directly approximated as a log-normal random variable [15]. But if we apply conditional probability to (6), it can be rewritten as

$$
\begin{aligned}
q^{*}= & \operatorname{Pr}\left\{Z_{t v}^{i, x}<0\right\} \operatorname{Pr}\left\{\bar{P}_{s u}^{i, x *} \leq \frac{Z_{t v}^{i, x}}{G_{a}^{i}} \mid Z_{t v}^{i, x}<0\right\} \\
& +\operatorname{Pr}\left\{Z_{t v}^{i, x} \geq 0\right\} \operatorname{Pr}\left\{\bar{P}_{s u}^{i, x *} \leq \frac{Z_{t v}^{i, x}}{G_{a}^{i}} \mid Z_{t v}^{i, x} \geq 0\right\} .
\end{aligned}
$$

Since $\bar{P}_{s u}^{i, x *}$ is non-negative, $\operatorname{Pr}\left\{\bar{P}_{s u}^{i, x *} \leq \frac{Z_{t v}^{i, x}}{G_{a}^{i}} \mid Z_{t v}^{i, x}<0\right\}=$ $\operatorname{Pr}\left\{\bar{P}_{s u}^{i, x *} \leq 0\right\}=0$. Hence we have

$q^{*}=0+q_{1}^{i, x} \operatorname{Pr}\left\{\bar{P}_{s u}^{i, x *} \leq \frac{Z_{t v}^{i, x}}{G_{a}^{i}} \mid Z_{t v}^{i, x} \geq 0\right\}, \quad \forall x \in X, \forall i \in \Lambda_{X}^{t v}$. 
Now we can approximate $Z^{\prime}=\left.Z_{t v}^{i, x}\right|_{Z_{t v}^{i, x} \geq 0}$ by a log normal random variable $\hat{Z}^{\prime} \sim L N\left(\mu_{\hat{Z}^{\prime}}, \sigma_{\hat{Z}^{\prime}}\right)$ by using method of moment [14].

With the above approximation, we can now convert the constraint (12) into dB domain

$$
q_{2}^{i, x} \approx q_{1}^{i, x} \operatorname{Pr}\left\{\bar{P}_{s u(\mathrm{dBm})}^{i, x *} \leq Z_{(\mathrm{dBm})}^{\prime}-\widehat{G}_{a(\mathrm{~dB})}^{i}\right\} \geq q * .
$$

Noting that both $Z_{(\mathrm{dBm})}^{\prime}$ and $\widehat{G}_{a(\mathrm{~dB})}^{i}$ follow normal distribution, we can solve the equivalent permissible transmit power as

$$
\begin{aligned}
\bar{P}_{s u(\mathrm{dBm})}^{i, x *}= & \mu_{\widehat{Z}^{\prime}(\mathrm{dBm})}-\mu_{\widehat{G}_{a}^{i}(\mathrm{~dB})} \\
& -\sqrt{2} \operatorname{erfc}^{-1}\left[2\left(1-\frac{q *}{q_{1}^{\prime}}\right)\right] \sqrt{\sigma_{\widehat{Z}^{\prime}(\mathrm{dB})}^{2}+\sigma_{\widehat{G}_{a}^{i}(\mathrm{~dB})}^{2}}
\end{aligned}
$$

where $\operatorname{erfc}^{-1}(\cdot)$ is the inverse complementary error function. One SU transmitting channel, $y$, can be adjacent to different TV broadcasting channels, and have different constraints. But the permissible power is always limited by the TV channel most vulnerable to ACI. Thus the permissible transmit power for each unoccupied channel $y$ is then given by

$$
P_{s u}^{i, y *}=\min _{x \in X}\left(\frac{\bar{P}_{s u}^{i, x *}}{\gamma\left(\Delta f_{x-y}\right)}\right) .
$$

It is worth mentioning that, the permissible transmit power can be decided by each SU independently following the proposed procedure, requiring only the knowledge about the secondary user density, deployment scenario and the TV coverage quality at its own location. It is not necessary to know the exact locations of $\mathrm{TV}$ receivers.

\section{Numerical Results}

In this section we first look into a test scenario to verify the proposed approach against simulations. Later, we applied the proposed procedure to obtain the spatial distribution of the permissible transmit power in a real-world environment for short-range secondary system.

\section{A. Verification of the Proposed Approach}

In the test scenario, we focus on a single pixel $i$ located at $D \mathrm{~km}$ away from the TV transmitter. The SUs are deployed in the pixel $i$ and its surroundings, following Poisson spatial distribution with density $\lambda^{i}$. Here we assume the studied pixel has 0.99 location probability without secondary interference.

In order to have a fair comparison with the reference geometry (Ref Geo) approach for multiple secondary transmitters described in [8], here we also considered a suburban environment with outdoor SU and rooftop TV antenna. Propagation model ITU Recommendation P.1411 [16] for suburban area over rooftop link is adopted for the distance based pathloss $g_{r}(r)$. It follows the free-space pathloss for line-of-sight distance up to $d_{\mathrm{LoS}}$, and changes to a higher pathloss exponent after a certain breakpoint. This breakpoint distance is set to be larger than the reference distance, $d_{\text {ref }}$, used in [8], so that the secondary interference is not underestimated in our model. The minimum separation distance $d_{0}$ between the SU transmitter and PU receiver is only limited by the physical difference in their antenna heights. On the other hand, we also modified the pathloss model such that $g_{r}(d)=g_{r}\left(d_{\text {ref }}\right)$, for $d \leq d_{\text {ref }}$, because it is assumed in [8] that the highest interfering link gain is achieved at $d_{\text {ref }}$.

The parameters for the test scenario are summarized in Table I. Only shadow fading is considered in this paper, as the wideband orthogonal frequency division multiplexing (OFDM) signal, used by the TV system and presumably also by the secondary system, is less prone to severe fast fading.

TABLE I: Simulation Parameter

\begin{tabular}{|l|l|}
\hline Parameter & Value \\
\hline TV signal standard deviation & $4.65 \mathrm{~dB}$ \\
\hline TV SINR requirement & $17.4 \mathrm{~dB}$ \\
\hline TV receiver sensitivity & $-80.6 \mathrm{dBm}$ \\
\hline TV receiver antenna height & $10 \mathrm{~m}$ at rooftop \\
\hline TV receiver antenna directivity & Defined by ITU-R BT419-3 [10] \\
\hline Cluster height & $10 \mathrm{~m}$ \\
\hline Location Probability Threshold $q *$ & 0.95 \\
\hline SU Transmitter height & $1.5 \mathrm{~m}$ \\
\hline SU Transmitter antenna gain & $0 \mathrm{dBi}$ \\
\hline $\begin{array}{l}\text { Secondary interference standard } \\
\text { deviation }\end{array}$ & $3 \mathrm{~dB}$ for $d \leq d_{L o S}$ and $6 \mathrm{~dB}$ for \\
\hline $\begin{array}{l}\text { Dominant interference region ra- } \\
\text { dius } R_{\varepsilon}\end{array}$ & $500 \mathrm{~m}$ for $\varepsilon=0.005$ \\
\hline $\begin{array}{l}\text { Line of Sight distance in ITU- } \\
\text { P1411 } d_{L o S} S\end{array}$ & $50 \mathrm{~m}$ \\
\hline Reference distance $d_{r e f}$ & $22 \mathrm{~m}$ \\
\hline Minimum separation distance $d_{0}$ & $8.5 \mathrm{~m}$ \\
\hline Geo-location database resolution & $250 \mathrm{~m} \times 250 \mathrm{~m}$ \\
\hline
\end{tabular}

A pair of the verification results are shown in Fig.3 and Fig.4, where Monte Carlo simulation is performed to adjust the maximum SU transmit power iteratively until the protection requirement is satisfied. As we can see from Fig.3, the proposed method slightly underestimate the permissible transmit power when the SU density is low. On the other hand, the estimated power level matches closely with the simulation result at higher SU density. The proposed method can always provide sufficient PU protection as seen in Fig.4. In comparison, the reference geometry method is overly pessimistic, even in the case with very high SU density.

\section{B. A Case Study: Permissible Transmit Power in Stockholm Area}

Having verified the approximations, we now apply this method to a real environment, utilizing the population density [17] and terrain elevation information [18]. The initial study is focused on the Stockholm area (Fig.5a). Here we assume the active SU density is one tenth of the population density and the SUs can select all the unoccupied TV channels with equal probability. There are one major TV transmitter (Tower A) with 280 meters height mast and $80 \mathrm{dBm}$ equivalent isotropically radiated power (EIRP), and a smaller repeater station (Tower B) with 90 meters height and $55 \mathrm{dBm}$ EIRP. Both transmitters are broadcasting on the same set of channels: channel 23, 42, 50, 53, 55, 56 and 59 [19].

Here a conservative assumption is made that, the cluster height and TV receiver antenna deployment throughout the 


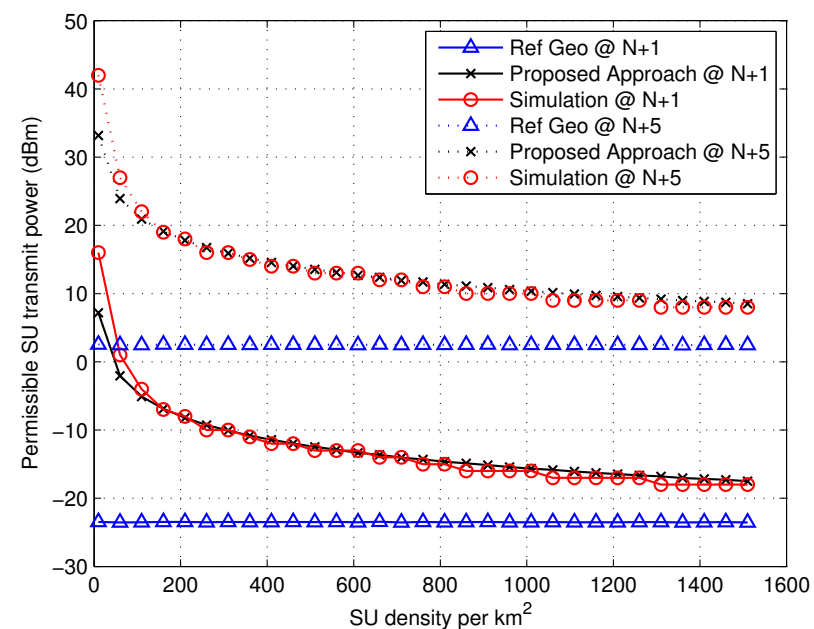

Fig. 3: Permissible transmit power with different SU densities

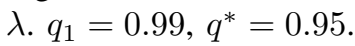

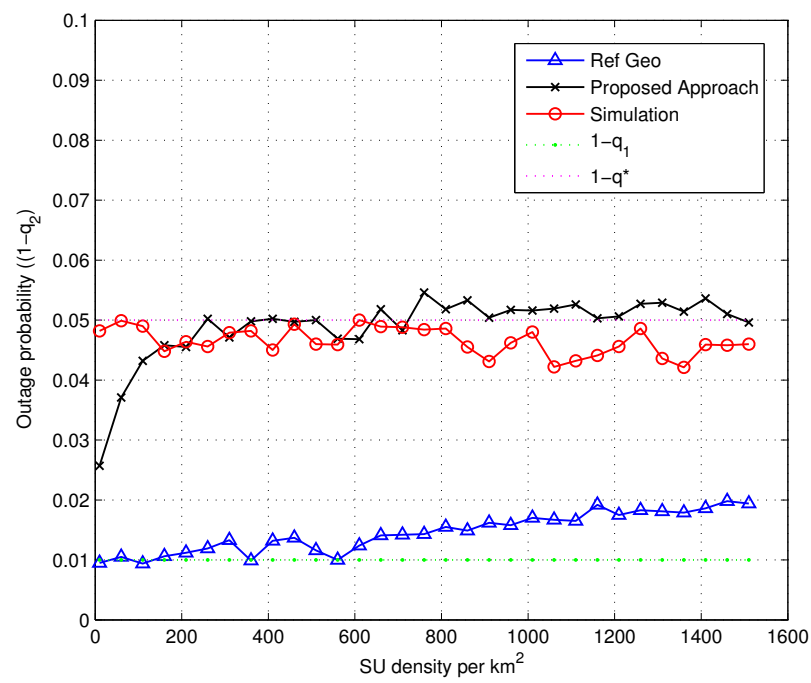

Fig. 4: TV outage probability with different SU densities $\lambda$. $q_{1}=0.99, q^{*}=0.95$.

studied area are the same as the suburban case defined in IV-A, in order to avoid any discontinuity may be caused by using different propagation models at different locations. The TV coverage (Fig.5b) is obtained by using ITU Recommendation P.1546 [20].

Fig.6 depicts the permissible transmit power per SU in Stockholm area on channel 51 (the first adjacent channel to channel 50, which is occupied by the TV broadcasting system in this area). We can see that most area in Stockholm permit more than $10 \mathrm{dBm} \mathrm{SU}$ transmit power, except a fraction of the dense urban area where the permissible transmit power is reduced to around $0 \mathrm{dBm}$. Similar calculation can be repeated for other adjacent channels as well, which usually permit higher secondary transmit power than the first adjacent

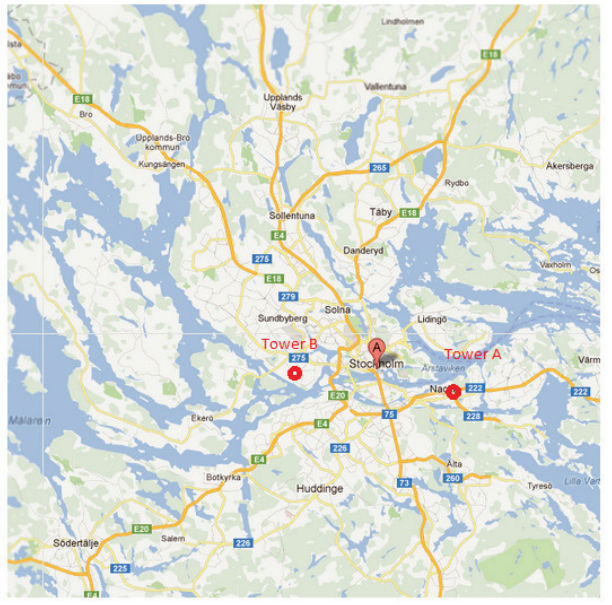

(a) Map of Stockholm area

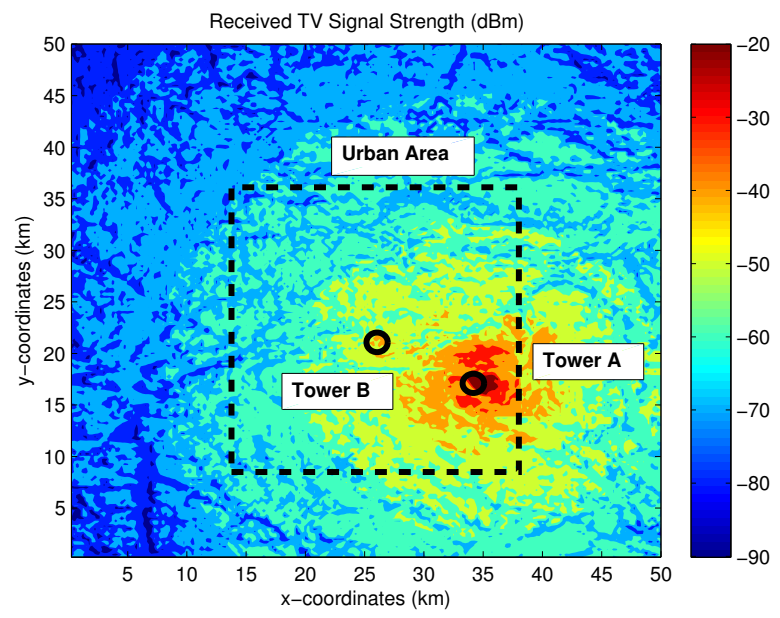

(b) Median received TV signal strength $(\mathrm{dBm})$ on channel 50

Fig. 5: Studied area in Stockholm.

channel.

\section{CONCLUSION}

In this paper, we investigated the adjacent channel interference problem in secondary access to TV white space. A statistical approach based on geo-location database is proposed to determine the permissible secondary transmit power under aggregate adjacent channel interference constraint. The proposed approach considers many aspects of the secondary interference, such as, shadow fading, TV receiver antenna directivity, the random deployment of secondary users and the cumulative effect of interferences from multiple adjacent channels. The complexity for computing the permissible secondary transmit power is, however, reduced considerably by the log-normal approximation of the secondary interference. The numerical results show that, this statistical approach 


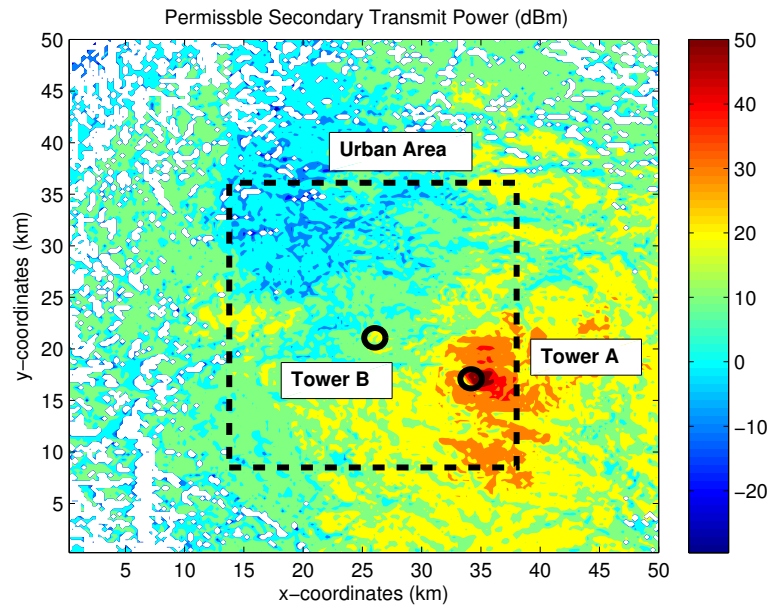

Fig. 6: Permissible transmit power on the first adjacent channel to a broadcasting channel in Stockholm area with $q *=0.95$.

predicates much higher permissible transmit power than the existing deterministic framework, while providing a reliable primary user protection. Furthermore, this approach can be easily applied to the real-world scenarios. A sample analysis for Stockholm area indicates considerable potential for shortrange secondary access in TV white space.

In the future study, we will consider the effect of external co-channel secondary interference and the possible ways to combine different propagation models used for different environments, so that we can provide a comprehensive analysis of the TV white space availability.

\section{ACKNOWLEDGMENT}

The research leading to these results has received partial funding from the European Union's Seventh Framework Programme FP7/2007-2013 under grant agreement $n^{\circ} 248303$ (QUASAR). The authors also would like to acknowledge the VINNOVA project MODyS for providing partial funding.

\section{REFERENCES}

[1] M. Nekovee, "Quantifying the Availability of TV White Spaces for Cognitive Radio Operation in the UK," in IEEE International Conference on Communications Workshops, June 2009.

[2] Federal Communications Commission, "In the Matter of Unlicensed Operation in the TV Broadcast Bands: Second Memorandum Opinion and Order," Sept. 2010. [Online] Available: http://www.fcc.gov,

[3] ECC Report 159, "Technical and Operational Requirements for the Possible operation of Coginitive Radio Systems in the White Spaces of the Frequency Band 470-790mhz". Jan. 2011, [Online]. Available: http://www.ero.dk.

[4] Ofcom, "Digital Dividend: Geolocation for Cognitive Access," Nov. 2009, [Online]. Available: http://stakeholders.ofcom.org.uk/consultations/cogaccess/.

[5] K. Ruttik, K. Koufos, and R. Jäntti, "Modeling of the Secondary System's Generated Interference and Studying of Its Impact on the Secondary System Design ," Radio Engineering, vol. 18, no. 7, pp. 1271-1278, Dec. 2010.

[6] Y. Selén, J. Kronander "Optimizaing Power Limits for White Space Devices under a Probability Constraint on Aggregated Inteference," submitted to IEEE International Symposium on Dynamic Spectrum Access Networks (DySPAN), 2012.
[7] E. Obregon, L. Shi, J. Ferrer, and J. Zander, "A model for Aggregate Adjacent Channel Interference in TV White Space," in IEEE 73rd Vehicular Technology Conference (VTC), May 2011.

[8] European Broadcasting Union, "The Cumulative Effect of Multiple WSD Interference," Sept. 2011, [Online]. Available: http://www.cept.org/meeting-documents.

[9] H. Karimi, "Geolocation Databases for White Space Devices in the UHF TV Bands: Specification of Maximum Permitted Emission Levels," in IEEE Symposium on New Frontiers in Dynamic Spectrum Access Networks (DySPAN), May 2011.

[10] "Directivity and Polarization Discrimination of Antennas in the Reception of Television Broadcasting," ITU-R Recommendation BT.419-3.

[11] M. Aljuaid and H. Yanikomeroglu, "Identifying Boundaries of Dominant Regions Dictating Spectrum Sharing Opportunities for Large Secondary Networks," in IEEE 21st International Symposium on Personal Indoor and Mobile Radio Communications (PIMRC), 2010 , Sept. 2010.

[12] A. Ghasemi and E. Sousa, "Interference Aggregation in SpectrumSensing Cognitive Wireless Networks," IEEE Journal of Selected Topics in Signal Processing, , vol. 2, no. 1, pp. 41 -56, Feb. 2008.

[13] M. Aljuaid and H. Yanikomeroglu, "A Cumulant-Based Characterization of the Aggregate Interference Power in Wireless Networks," in IEEE 71st Vehicular Technology Conference (VTC) , May 2010.

[14] K. W. Sung, M. Tercero, and J. Zander, "Aggregate Interference in Secondary Access with Interference Protection," IEEE Communications Letters, vol. 15, no. 6, pp. 629 -631, June 2011.

[15] SE43 Meeting Document, "On the Permissible Transmit Power for WSDs in TV White Space," Sept. 2011, [Online]. Available: http://www.cept.org/meeting-documents.

[16] "Propagation Data and Prediction Methods for the Planning of ShortRange Outdoor Radio Communication Systems and Radio Local Area Networks in the Frequency Range $300 \mathrm{MHz}$ to $100 \mathrm{GHz}$,' ITU Recommendation P.1411.

[17] Center for International Earth Science Information Network (CIESIN), Columbia University, and Centro Internacional de Agricultura Tropical (CIAT), “Gridded Population of the World, version 3,"Nov. 2011, [Online]. Available: http://sedac.ciesin.columbia.edu/gpw.

[18] Centro Internacional de Agricultura Tropical (CIAT), "SRTM Digital Elevation Database,"Nov. 2011, [Online]. Available: http://srtm.csi.cgiar.org.

[19] Post och Telestyrelsen, "Tillst?dsdata Digital TV Mars 2008 Version A," Mar. 2008, [Online]. Available: http://www.pts.se/sv/Dokument/.

[20] "Method for Point-to-Area Predictions for Terrestrial Services in the Frequency Range $30 \mathrm{MHz}$ to $3000 \mathrm{MHz}$," ITU Recommendation P.1546-4. 